\title{
Two-dimensional random tilings of large codimension: new progress
}

\author{
N. Destainville ${ }^{\mathrm{a}, *}$, M. Widom ${ }^{\mathrm{b}}$, R. Mosseri ${ }^{\mathrm{c}}$, F. Bailly $^{\mathrm{d}}$ \\ ${ }^{a}$ Laboratoire de Physique Quantique-UMR 5626, Université Paul Sabatier, 31062 Toulouse Cedex 04, France \\ ${ }^{\mathrm{b}}$ Department of Physics, Carnegie Mellon University, Pittsburgh, PA 15213, USA \\ ${ }^{\mathrm{c}}$ Groupe de Physique du Solide, Tour 23, 5e étage, Universités Paris 7 et 6, 2 place Jussieu, 75251 Paris Cedex 05, France \\ ${ }^{\mathrm{d}}$ Laboratoire de Physique du Solide-CNRS, 1 place Aristide Briand, 92195 Meudon Cedex, France
}

Received 13 September 1999; accepted 20 October 1999

\begin{abstract}
Two-dimensional random tilings of rhombi can be seen as projections of two-dimensional membranes embedded in hypercubic lattices of higher dimensional spaces. Here, we consider tilings projected from a $D$-dimensional space. We study the limiting case, when the quantity $D$, and therefore the number of different species of tiles, become large. We had previously demonstrated [M. Widom, N. Destainville, R. Mosseri, F. Bailly, in: Proceedings of the Sixth International Conference on Quasicrystals, World Scientific, Singapore, 1997.] that, in this limit, the thermodynamic properties of the tiling become independent of the boundary conditions. The exact value of the limiting entropy and finite $D$ corrections remain open questions. Here, we develop a mean-field theory, which uses an iterative description of the tilings based on an analogy with avoiding oriented walks on a random tiling. We compare the quantities so-obtained with numerical calculations. We also discuss the role of spatial correlations. (C) 2000 Elsevier Science B.V. All rights reserved.
\end{abstract}

Keywords: Two-dimensional random tilings; Entropy; Mean-field theory

\section{Introduction}

The understanding of the stability of quasicrystalline materials, discovered about 15 years ago [2], motivates the study of random tilings. Indeed, it is believed that in these materials, atomic motifs form geometric tiles; the random tiling hypothesis states that these tiles can be rearranged via local degrees of freedom, which gives access to a finite configurational entropy which is supposed to favor the quasicrystalline symmetry against other competitive crystalline phases [3,4]. Indeed, such symmetries, a priori inherent to Penrose-like structures [5,6], are still displayed by their random counterpart, which are therefore, good candidates to model quasicrystalline materials [7]. The best description for real quasicrystals remains an open question.

Two years ago, we presented initial results on twodimensional random tilings of large codimension [1] in which, as had been anticipated by Henley [4] analytic approach is more likely to be developed than in the finite-dimensional case. The present paper contains preliminary results in the understanding of these systems. We developed a mean-field theory, based upon an iterative

\footnotetext{
* Corresponding author. Tel: +33-5-61-55-60-48; fax: +33-5-61-55-60-48.

E-mail address: destain@irsamc2.ups-tlse.fr (N. Destainville).
}

walk-on-tiling construction of large codimension tilings, providing a new insight into this infinite codimension limit. Except for the diagonalization of an operator, all this work relies on exact calculations. The results so-obtained are validated by Monte Carlo simulations, based upon the same iterative process. A more complete and explicit presentation will be published elsewhere [8].

In the two following sections, we briefly define what infinite codimension tilings are and we recall results from reference [1]. In Section 4, we explain the mean-field theory and we compare it to results of Monte Carlo simulations.

\section{Model}

We define two-dimensional random tilings of rhombi, denoted as $D \rightarrow 2$ tilings, as projections from a $D$-dimensional cubic lattice into a two-dimensional plane. Facets of the cubic lattice project into rhombi [9-11,17]. The projection is chosen so that the rhombi have unit edge length and their angles are multiple of $\pi / D$. As a consequence, the tiles are arranged in tilings with statistical $2 D$-fold rotational symmetry. Fig. 1 displays such tilings. In this case, they fill regular $2 D$-gons. Such polygonal boundary conditions will be called fixed in the following. We will also consider free boundary tilings in this paper. In the de Bruijn grid 

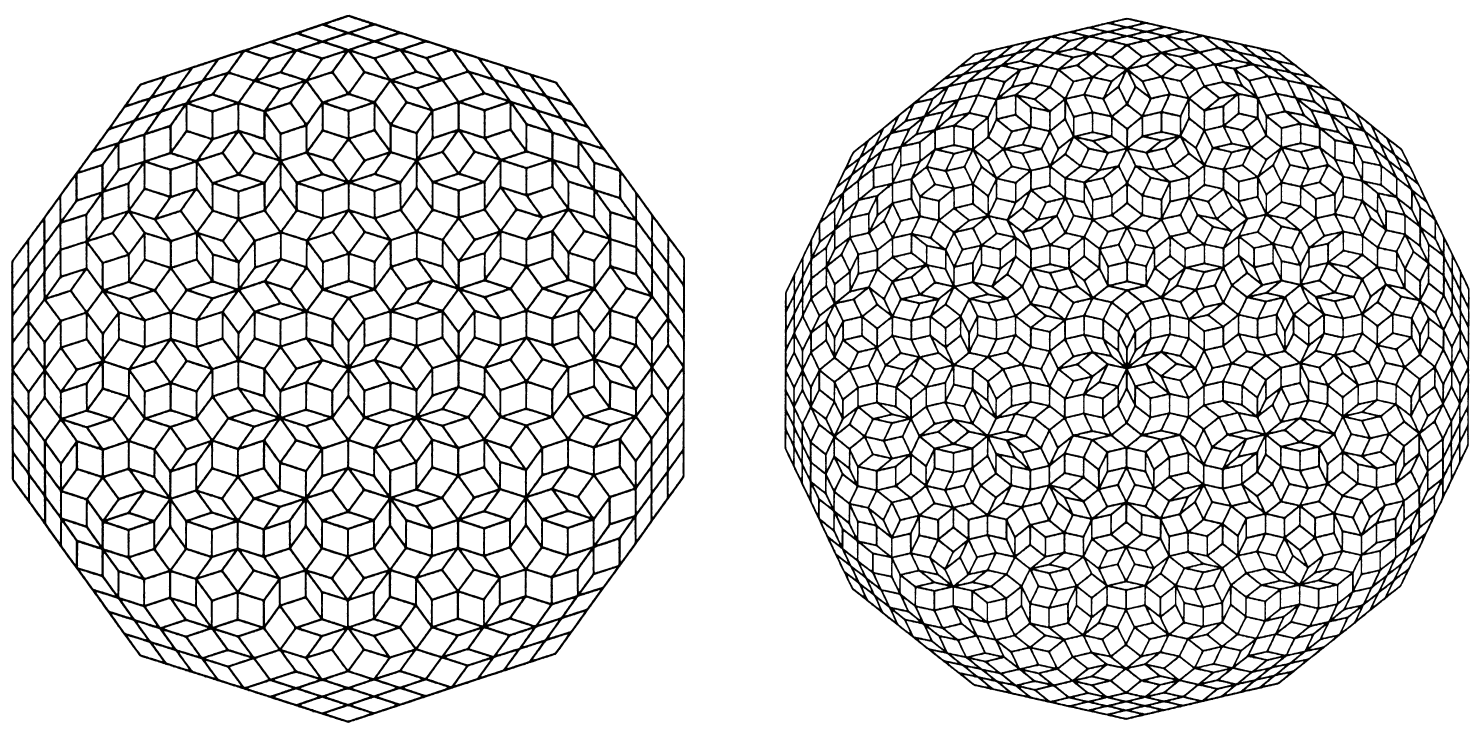

Fig. 1. Two examples of fixed boundary tilings for $D=5$ and 7. In this case, the side lengths of the $2 D$-gons are all equal to $p=8$.

representation [12-14,18], any two lines of different orientation cross in the fixed boundary case, whereas they do not necessarily cross in the free boundary case.

Before going on, let us mention that the effect of boundaries was recently studied $[15,19,20]$ and turned out to be highly non trivial: fixed boundaries have macroscopic effects on tilings at the infinite size limit, which are characterized by a gradient of entropy between the boundary and the center of the tilings. As a consequence, fixed boundary tilings have a lower entropy than free boundary ones. Note that periodic boundary conditions give the same entropy as free ones at the infinite size limit [16], and that this entropy only depends on the relative fractions of tiles and not on the sample shape.

\section{Thermodynamic limit}

We demonstrated previously [1] that when the codimension ${ }^{1}$ of the plane tilings becomes large, the entropy difference between fixed and free boundary conditions vanishes. More precisely, the structural inhomogeneity between regions close to the boundary and the bulk observed in finite codimension tilings tends to disappear. Moreover, it was argued that this limiting entropy is still valid when the side length $p$ of the polygonal boundary remains finite as the codimension goes to infinity. In particular, one can choose $p=1$ in order to perform more simple calculations [1]. In this reference, an upper bound of the limit entropy, $\sigma<\log 2$, was also given.

\footnotetext{
${ }^{1}$ That is the difference $D-2$ between the cubic lattice dimension $D$ and the tiling dimension 2 .
}

\section{Mean-field theory}

Both the mean-field theory and the numerical calculations presented below rely on an iterative construction of large codimension tilings, based upon the de Bruijn representation of tilings $[12,18]$. This point of view can be used either in the fixed boundary context [1] or in the free boundary one.

\subsection{Walk-on-tiling algorithm}

As illustrated in Fig. 2, a $D \rightarrow 2$ tiling can be seen as a collection of non-intersecting paths chosen on a $D-1 \rightarrow 2$ random tiling. These paths are directed from bottom to top. They follow the edges of the $D-1 \rightarrow 2$ underlying tiling. Then these paths are 'opened' along a new edge direction in order to form the $D$-th de Bruijn family and then a $D \rightarrow 2$ tiling. Conversely, de Bruijn lines of family $D$ in a $D \rightarrow 2$ tiling can be collapsed into paths on a $D-1 \rightarrow 2$ tiling. As a consequence, there is a one-to-one correspondence between $D \rightarrow 2$ tilings with $p$ de Bruijn lines in family $D$ and collections of $p$ non-intersecting directed paths on $D-1 \rightarrow 2$ tilings.

\subsection{Mean-field theory and Monte Carlo simulations}

As we explained above, as $D$ becomes large, the enumeration problem can be simplified into a problem with a single line in each de Bruijn family. In other words, de Bruijn lines of the same family do not interact, and the number of $p$-line configurations can be factorized into a product of independent single-line configurations. As a consequence, the entropy is equal to

$\sigma=\lim _{D \rightarrow \infty} \lim _{k \rightarrow \infty} \frac{\log N_{D}(k)}{k}$, 

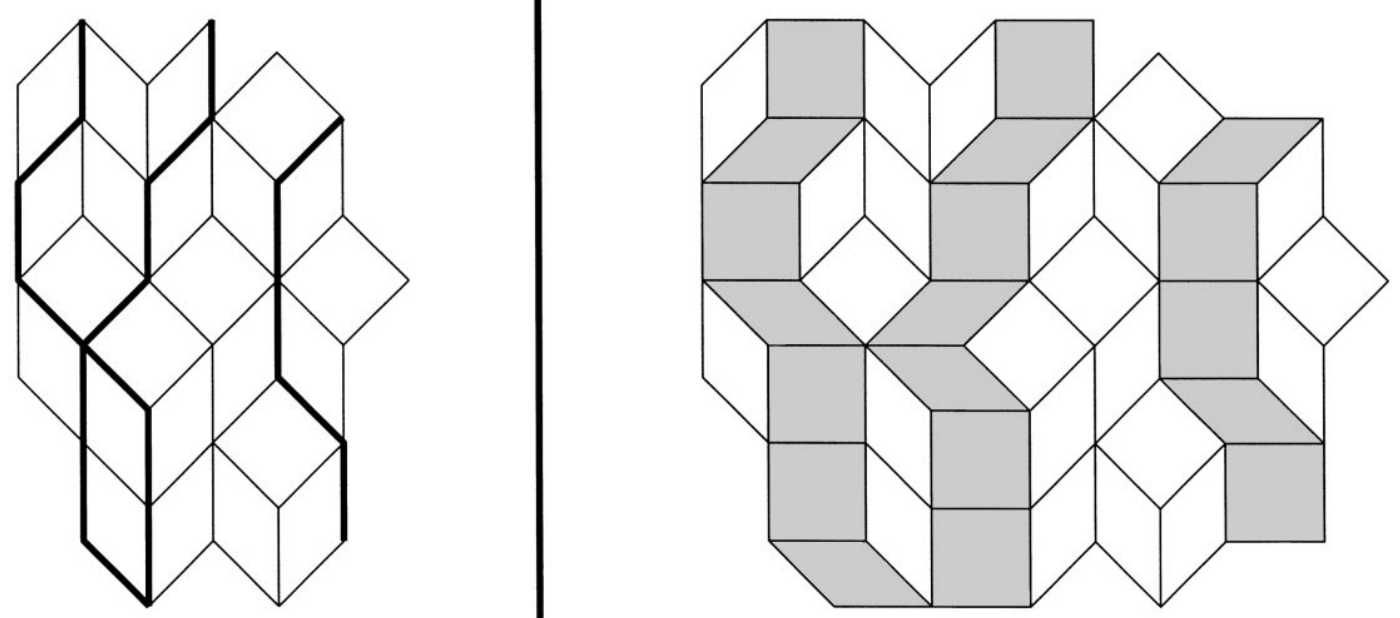

Fig. 2. Iterative process for the construction of $D \rightarrow 2$ tilings: paths are chosen on a $D-1 \rightarrow 2$ tiling (left), and are "opened" in a new direction to form de Bruijn lines of the $D$-th family (right).

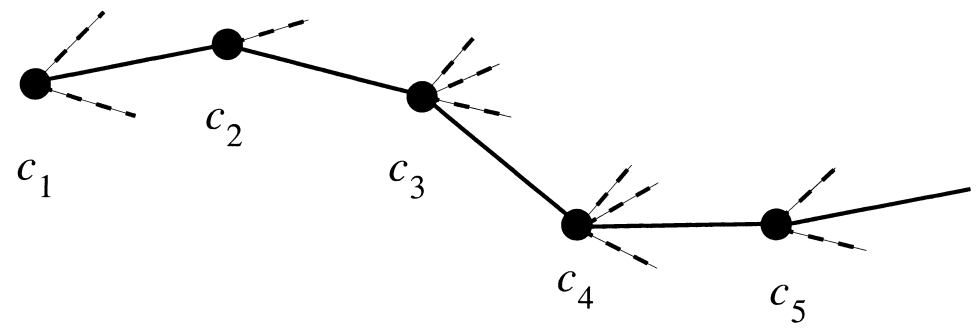

Fig. 3. A random path on a tiling as a succession of uncorrelated vertices (the bottom-to-top path has been rotated into a left-to-right path for sake of convenience).

where $N_{D}(k)$ is the number of $k$-step paths on a $D$-dimensional random tiling $[1,8]$.

Now, the mean-field approximation assumes that the steps of such paths are uncorrelated. Then the number of paths reads

$N_{D}(k) \simeq \prod_{j=1}^{k} c_{j}$,

where $c_{j}$ are the numbers of choices to be made at each vertex $j$ to add a new step to the path (see Fig. 3).
Keep in mind that, even if they are uncorrelated, the vertices $j$ belong to a random $D \rightarrow 2$ tiling. Therefore, the numbers of choices $c_{j}$ are distributed according to a probability distribution $\pi_{D}(c)$. When $D$ tends to infinity, this distribution tends toward a limiting distribution, denoted by $\pi(c)$. Thus, after a short calculation, Eqs. (1) and (2) become:

$\sigma=\sum_{c=1}^{\infty} \pi(c) \log c$.
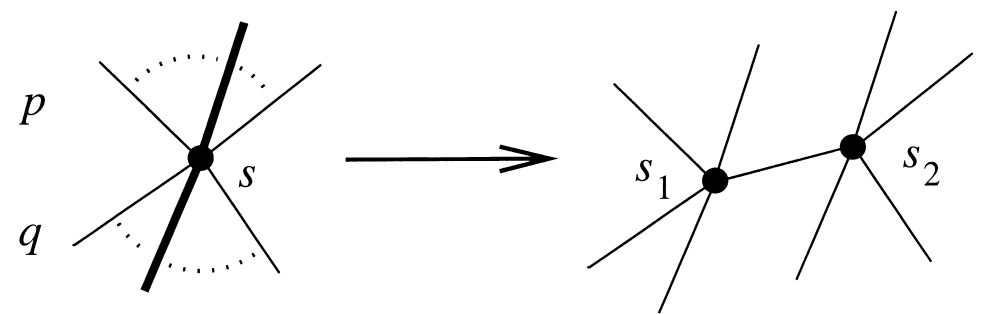

Fig. 4. A path going through a vertex $s$ of a $D \rightarrow 2$ tiling gives birth to two vertices $s_{1}$ and $s_{2}$ of a $D+1 \rightarrow 2$ tiling. 
Table 1

The first values of the limit distribution $\pi(c)$, obtained both in the mean-field approximation and numerically by Monte Carlo simulations

\begin{tabular}{lllll}
\hline$c$ & 1 & 2 & 3 & 4 \\
\hline Mean-field & 0.30 & 0.45 & 0.19 & 0.04 \\
Numerical & 0.26 & 0.51 & 0.20 & 0.03 \\
\hline
\end{tabular}

To get the mean-field distribution $\pi(c)$, let us first denote by $\pi_{D}(q, p)$ the fraction of vertices on a $D \rightarrow 2$ random tiling with $q$ incoming edges ('legs') and $p$ outgoing ones ('arms') (see Fig. 4) The distributions $\pi_{D}(c)$ and $\pi(c)$ will easily be derived from $\pi_{D}(q, p)$. If no correlations are taken into account, a (q,p)-vertex $s$ of a $D \rightarrow 2$ tiling will be visited by the paths with a probability $\pi_{D}(q, p)$ and will then give birth to two vertices $s_{1}$ and $s_{2}$, as illustrated in Fig. 4 These latter vertices belong to a $D+1 \rightarrow 2$ tiling. Each leg and each arm of $s$ will be chosen with probabilities $1 / q$ and $1 / p$, respectively. As a consequence, in this mean-field approximation, the probabilities $\pi_{D+1}(q, p)$ can be written as linear combinations of the probabilities $\pi_{D}(q, p)$. The corresponding linear operator will be denoted by $\mathcal{A}$. It is infinite-dimensional since $q$ and $p$ can be arbitrarily large when $D$ goes to infinity.

The limiting distribution $\pi(q, p)$ is the fixed point of $\mathcal{A}$, that is the eigenvector associated with the eigenvalue 1 . We have calculated this fixed point numerically, yielding the values of $\pi(c)$ listed in Table 1. The mean-field entropy (3) is then approximately equal to $\sigma=0.60$. The distribution $\pi(q, p)$ also provides the mean-field correlations between $p$ 's and $q$ 's: their covariance is $\operatorname{cov}(q, p) \simeq-0.44^{2}$.

Let us mention that we have not taken into account the fact that at the step $D$ of the iterative process, only a fraction of order $1 / D$ of the vertices are visited by paths [1]. This modification does not alter the fixed point of the process [8], but only the finite $D$ corrections: they have a power-law behavior instead of an exponential one.

To close this section, let us emphasize that these mean-field values are satisfyingly close to numerical values obtained by Monte Carlo simulations [8], as displayed in Table 1. The corresponding numerical entropy and correlations are approximately equal to 0.57 and -0.36 , respectively.

\section{Conclusion}

The above mean-field theory provides quite satisfying numerical results concerning the local structure of large codimension random tilings, in terms of vertex statistics distributions. As a consequence, it provides a good approximate value of the limit entropy of such systems.
To go further and to get better approximations, it will be necessary to include the role of spatial correlations in our calculations, by taking into account the distribution of tiling patches bigger than single vertices.

Moreover, it would be useful to get informations about finite $D$ corrections to the limit entropy $\sigma$, since finite codimensional systems are related to real quasicrystals. Two ingredients will be taken into account: the power-law corrections of the mean-field theory as discussed above, and the effects of contacts between de Bruijn lines, which tend to decrease the entropy [1].

\section{Acknowledgements}

This work was supported in part under a joint research program of the NSF and CNRS.

\section{References}

[1] M. Widom, N. Destainville, R. Mosseri, F. Bailly, in: Proceedings of the Sixth International Conference on Quasicrystals, World Scientific, Singapore, 1997.

[2] D. Shechtman, I. Blech, D. Gratias, J.W. Cahn, Phys. Rev. Lett. 53 (1984) 1951.

[3] V. Elser, Phys. Rev. Lett. 54 (1985) 1730.

[4] C.L. Henley, in: D.P. Di Vincenzo, P.J. Steinhart (Eds.), Quasicrystals, The State of the Art, World Scientific, Singapore, 1991.

[5] R. Penrose, Bull. Inst. Math. Appl. 10 (1974) 226.

[6] D. Levine, P.J. Steinhardt, Phys. Rev. Lett. 53 (1984) 2477.

[7] M. Widom, in: M.V. Jarić, S. Lundqvist (Eds.), Quasicrystals, World Scientific, Singapore, 1990.

[8] N. Destainville, F. Bailly, R. Mosseri, M. Widom, in preparation.

[9] M. Duneau, A. Katz, Phys. Rev. Lett. 54 (1985) 2688.

[10] A.P. Kalugin, A.Y. Kitaev, L.S. Levitov, JETP Lett. 41 (1985) 145.

[11] N. Destainville, R. Mosseri, F. Bailly, J. Stat. Phys. 87 (3/4) (1997) 697.

[12] N.G. de Bruijn, Ned. Akad. Wetensch. Proc. A84 (1981) 39.

[13] J.E.S. Socolar, P.J. Steinhardt, D. Levine, Phys. Rev. B 32 (8) (1985) 5547.

[14] F. Gähler, J. Rhyner, J. Phys. A: Math. Gen. 19 (1986) 267.

[15] N. Destainville, J. Phys. A: Math. Gen. 31 (1998) 6123.

[16] W. Li, H. Park, M. Widom, J. Stat. Phys. 66 (1992) 1.

[17] A.P. Kalugin, A.Y. Kitaev, L.S. Levitov, J. Phys. Lett. France 46 (1985) L601.

[18] N.G. de Bruijn, J. Phys. France 47 (1986) C3-9.

[19] H. Cohn, M. Larsen, J. Propp, New York J. Math. 4 (1998) 137.

[20] H. Cohn, R. Kenyon, J. Propp, preprint (www-math.mit.edu/ $\sim$ propp/variational.ps), 1998.

${ }^{2}$ As a matter of fact, this value seems to be equal to $-4 / 9$. 